\title{
Silica-Gelatin Hybrids with Tailorable Degradation and Mechanical Properties for Tissue Regeneration
}

By Oliver Mahony,* Olga Tsigkou, Claudia Ionescu, Caterina Minelli, Lowell Ling, Ruth Hanly, Mark E. Smith, Molly M. Stevens and Julian R. Jones*

[*] Dr. J. R. Jones, R. Hanly

Department of Materials, Imperial College London, SW7 2AZ, UK.

E-mail: julian.r.jones@imperial.ac.uk

$\left.{ }^{*}{ }^{*}\right] \quad$ O. Mahony, Dr. O. Tsigkou, Dr. C. Minelli, L. Ling, Prof. M. M. Stevens Department of Materials and Institute of Bioengineering, Imperial College London, SW7 2AZ, UK.

E-mail: oliver.mahony@imperial.ac.uk

C. Ionescu, Prof. M. E. Smith

Department of Physics, University of Warwick, Coventry, CV4 7AL, UK.

Keywords: Hybrid materials, Biomedical Applications, Tissue Engineering, Porous Materials, Silica, Composites

Nature has evolved mechanisms to create a diversity of specialised materials through nanoscale organisation. Inspired by nature, we have designed hybrid materials with highly tailorable properties, which are achieved through careful control of their nanoscale interactions. These novel materials, based on a silicagelatin hybrid system, have the potential to serve as a platform technology for human tissue regeneration. Strong chemical bonds between the inorganic and organic constituents of the hybrid are essential to enable the precise control of mechanical and dissolution properties. Furthermore, hybrid scaffold porosity was found to highly influence mechanical properties, to the extent where scaffolds of particular strength could be specified based on their porosity. We envisage these 
Submitted to

hybrid materials will find a diverse application in both hard and soft tissue

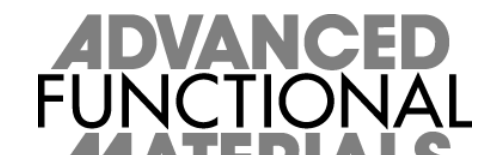
regenerating scaffolds.

\section{Introduction}

In an ageing population there is increased demand on our body parts, leading to an increased need to regenerate diseased or damaged tissues. ${ }^{[1,2]}$ Tissue engineering is currently a popular approach to this problem, however, biomaterials suitable for tissue engineering must meet some very challenging criteria. ${ }^{[3,4]}$ For instance they must provide a temporary template (scaffold) for tissue growth; exhibit similar mechanical properties to those of the host tissue; and resorb with controllable degradation rates. Research into silica based biomaterials are commonplace due to their biocompatibility ${ }^{[5]}$ and they have been utilised for bone regeneration strategies, ${ }^{[6-8]}$ for angiogenesis, ${ }^{[9,10]}$ drug delivery ${ }^{[11]}$ and in cements. ${ }^{[12]}$ However silica is brittle, which limits its application in tissue engineering. But if polymers are introduced to develop composites with silica there is the potential to create tough materials, of particular use in the area of bone regeneration. ${ }^{[13-15]}$ However, a drawback with many conventional composites is that the constituent phases interact on a micron scale, which can result in differential resorption rates during dissolution. This inevitably leads to material instability in vivo. Biomaterial composites with concurrent degradation of the organic and inorganic constituent phases are yet to be demonstrated. 


\section{Submitted to

In Hybrid materials, however, the constituent phases interact chemically on a nanoscale ${ }^{[16-20]}$ and we propose that this finer control of phase interaction can enable precise control of parameters such as degradation rates and mechanical properties. Here we present a platform technology based on a silica-gelatin hybrid material that demonstrates highly tailorable properties. Strong covalent links between the inorganic and organic chains are key to obtaining a tough composite material that degrades as one material.

\section{Results and discussion}

\subsection{A true hybrid material}

Gelatin was chosen as the toughening polymer as it is a hydrolysed form of collagen, which is a major constituent of the extracellular matrix of all tissues ${ }^{[21]}$ and as such recognisable and adhered to by many cell types. ${ }^{[22]}$ Gelatin is more practical than collagen from a materials processing point of view and was preferred over other popular resorbable polyesters such as poly lactic and glycolic acids as these degrade through a self catalysed hydrolysis process that can lead to poor control of dissolution characteristics in vivo. ${ }^{[23]}$ Silica gelatin conventional composites have been synthesised in the past using sol-gel techniques, ${ }^{[24-26]}$ but as non-functionalised gelatin is soluble in an in vivo environment it is essential to covalently link it to the silica phase in order to prevent premature dissolution and instability of the gelatin phase; hence we produced a true hybrid material. Previously researchers have functionalised gelatin using 3-glycidoxyproyltrimethoxysilane (GPTMS). ${ }^{[27,28]}$ 


\section{Submitted to

However, in these hybrid systems the silica contribution came from GPTMS, and therefore the inorganic proportion of the hybrid could not be varied independently of the degree of inorganic-organic coupling. Therefore the matrix of the hybrids was the organic phase, which may limit its maximum strength. In our study silica content was controlled independently of the degree of inorganic-organic coupling by using tetraethyl orthosilicate (TEOS) as a separate source of silica.

A sol-gel synthesis system was used, making use of GPTMS as a coupling agent to covalently link gelatin to silica. Initially, GPTMS was reacted with gelatin in dilute hydrochloric acid $(\mathrm{HCl})$. The epoxy ring of GPTMS opened and reacted with gelatin's carboxylic acid groups ${ }^{[27]}$ as shown in equation (1), where $\mathrm{R}$ represents a gelatin molecule.

1

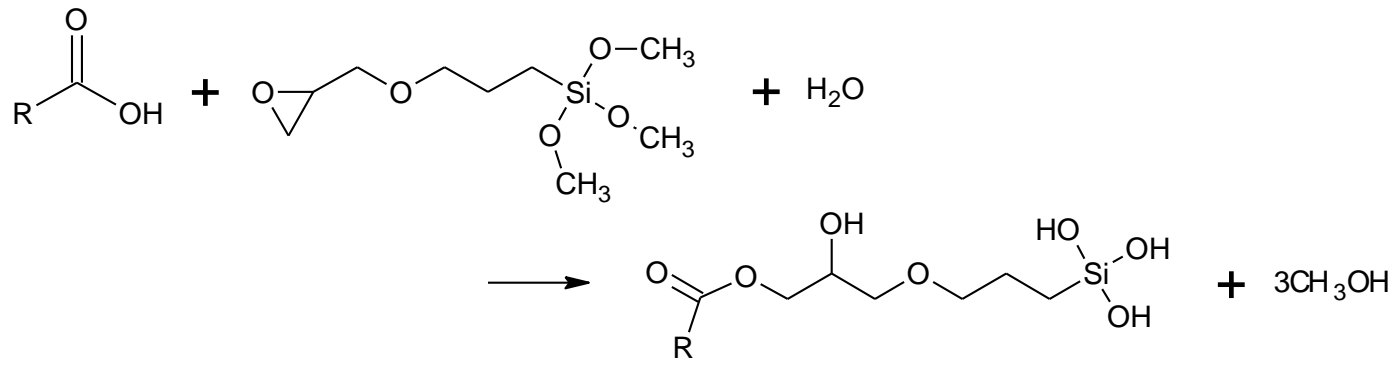

Simultaneously, hydrolysis of the methoxysilane groups occurred yielding silanol groups (Si-OH). TEOS, the sol-gel precursor for silica, was hydrolysed separately with aqueous $\mathrm{HCl}$. This resulted in a colloidal solution (a sol) of $\mathrm{Si}(\mathrm{OH})_{4}{ }^{[29]}$ as shown in equation (2). 


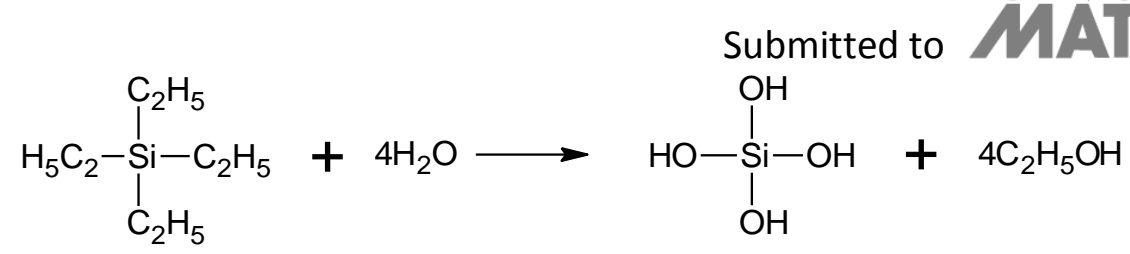

It was hypothesised that the silanol groups of the functionalised polymer would react to those of the hydrolysed TEOS when the solutions were mixed forming Si-OSi bonds through condensation. As condensation reactions continued the sol gelled due to the formation of more Si-O-Si bonds, creating a silica network covalently linked to gelatin as shown in equation $(3) . .^{[30,31]}$

3<smiles>CCCCCCCO[Si](O)(O)O[Si](O)(O)O</smiles>

Sols were cast into moulds, where they gelled as monoliths and were aged (left for a period of time before drying to allow for further condensation) and dried.

To test the hypothesis that GPTMS condensed into the silicate network linking the inorganic and organic chains, ${ }^{29}$ Si Magic Angle Spinning Nuclear Magnetic Resonance (MAS NMR) and atomic force microscopy (AFM) were employed. The 29Si MAS NMR spectra (Fig. 1a) can determine relative amounts of $Q$ and $T$ species in a hybrid. $Q$ species describe the connectivity of the silicate network, with a $Q^{4}$ species signifying a silicon atom bonded to four other silicon atoms via "bridging" oxygens (four network forming $\mathrm{Si}-\mathrm{O}-\mathrm{Si}$ bonds) and $\mathrm{Q}^{3}$ signifies three bridging oxygens and one "non-bridging" oxygen (typically Si-OH). T species are indicative of a silicon atom from a silicate network that is also bound to a carbon atom as shown in the example 


\section{Submitted to \\ FUNCTIONAL}

in Figure 1b. As the hybrid's molar ratio of GPTMS : gelatin (referred to here as Cfactor) increased, there was a notable increase in the T species in the NMR spectra, indicating an increase in inorganic-organic coupling. Therefore the NMR demonstrates that GPTMS was successful in providing a covalent link from the organic gelatin molecules to the inorganic silicate network, which is essential to the hybrid design. Quantification of the relative proportions of $T$ and $Q$ species (Fig. 1C) demonstrated that as C-factor increased the degree of condensation of the silicate network also increased as shown by the dominance of $\mathrm{T}^{3}$ and $\mathrm{Q}^{4}$ species of the hybrids with a C-factor of 1500. In fact, the total proportion of silicon atoms with no non-bridging oxygens increased from 61\% in C-factor 500 to $72 \%$ in C-factor 1500 hybrids. This reduction in non-bridging oxygens was confirmed with Fourier transform infrared spectroscopy (Fig. 2). Sol-gel hybrids have a nanoscale surface texture (Fig. 4a and b), which leads to a mesoporous structure (pores in the range 2$50 \mathrm{~nm})$. Reduced amounts of non-bridging oxygens associated with increased Cfactor lead to a material with a denser silicate network. The shape of isotherms, generated from nitrogen sorption (Type IV, Fig. 3a), were indicative of an interconnected mesoporous material (pores in the range of 2-50 nm). Pore size distributions (obtained by BJH analysis of the desorption branch of the isotherm) confirmed the reduction in non-bridging oxygens as the modal mesopore diameter was found to decrease as C-factor increased (Fig. 3b). For C-factors above 500 the pores become smaller than $2 \mathrm{~nm}$ (considered nanoporous, rather than mesoporous), which could not be measured accurately with the nitrogen adsorption instrument used. 

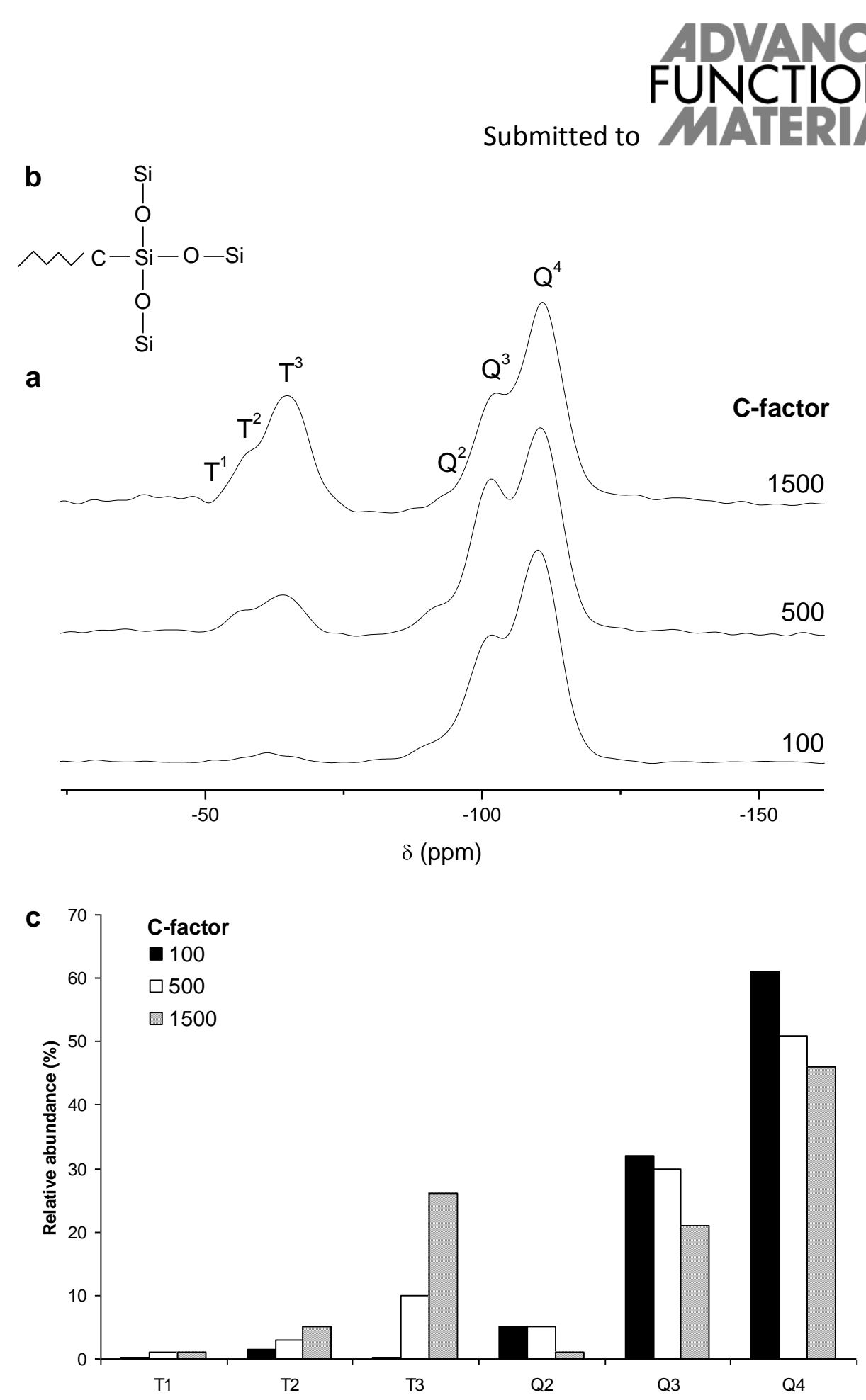

Figure 1. ${ }^{29}$ Si MAS NMR for hybrids (30 wt\% gelatin) of C-factor 100, 500 and 1500. a, Spectra with $T$ and $Q$ species indicated. $\mathbf{b}$, An example chemical structure of a silicon $\mathrm{T}^{3}$ species. $\mathrm{C}$, The relative abundance of the $\mathrm{T}$ and $\mathrm{Q}$ species quantified for all $\mathrm{C}$ factors (data acquired through deconvolution of peaks from spectra in a). 


\section{Submitted to \\ FUNCTIONAL}

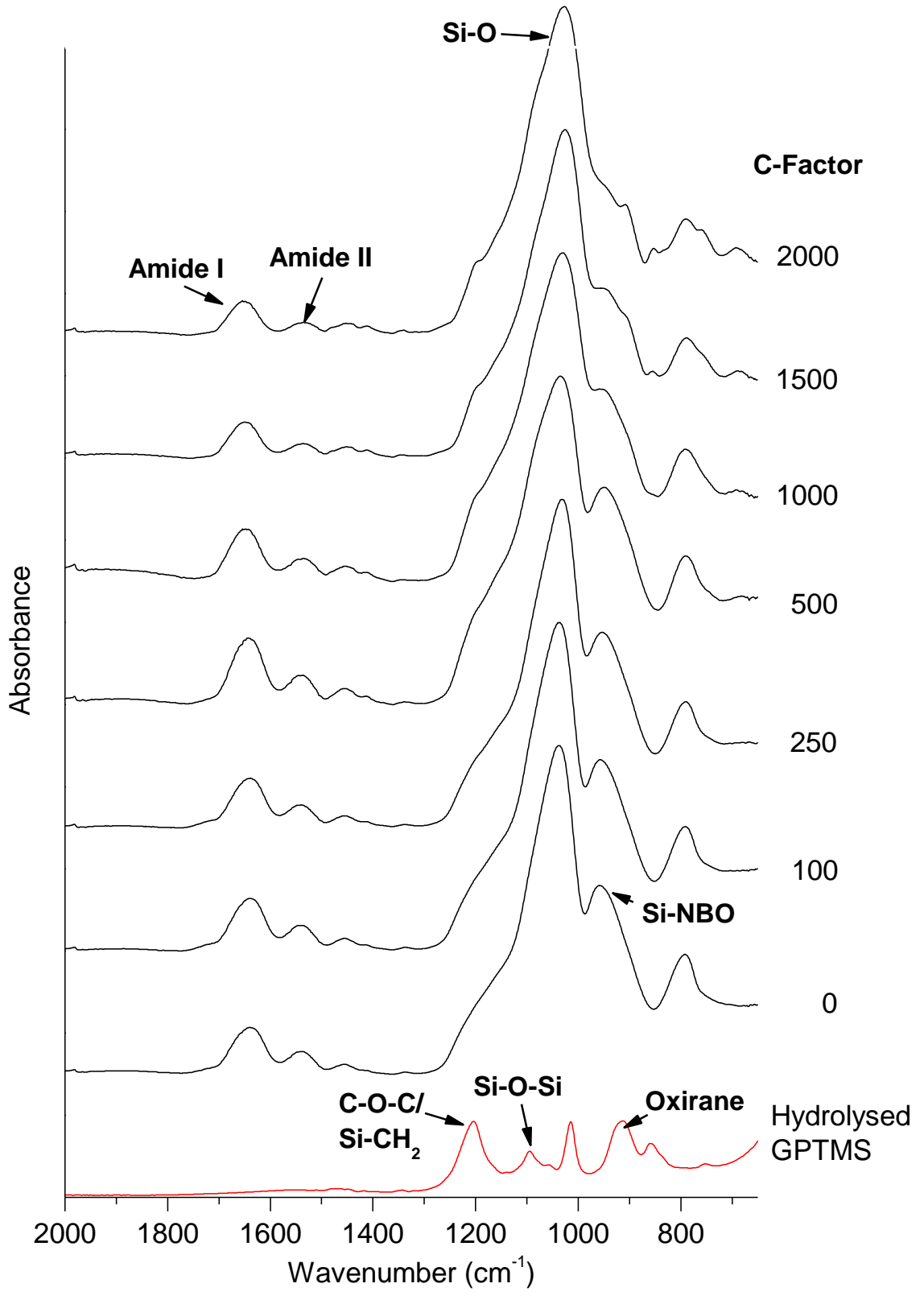

Figure 2. FTIR spectroscopy on silica-gelatin hybrids, C-factor 0-2000 (as indicated), and hydrolysed GPTMS (i.e. following reaction with deuterium oxide for $1 \mathrm{~h}$ ). Vibrational bands of note have been labelled. 


\section{Submitted to

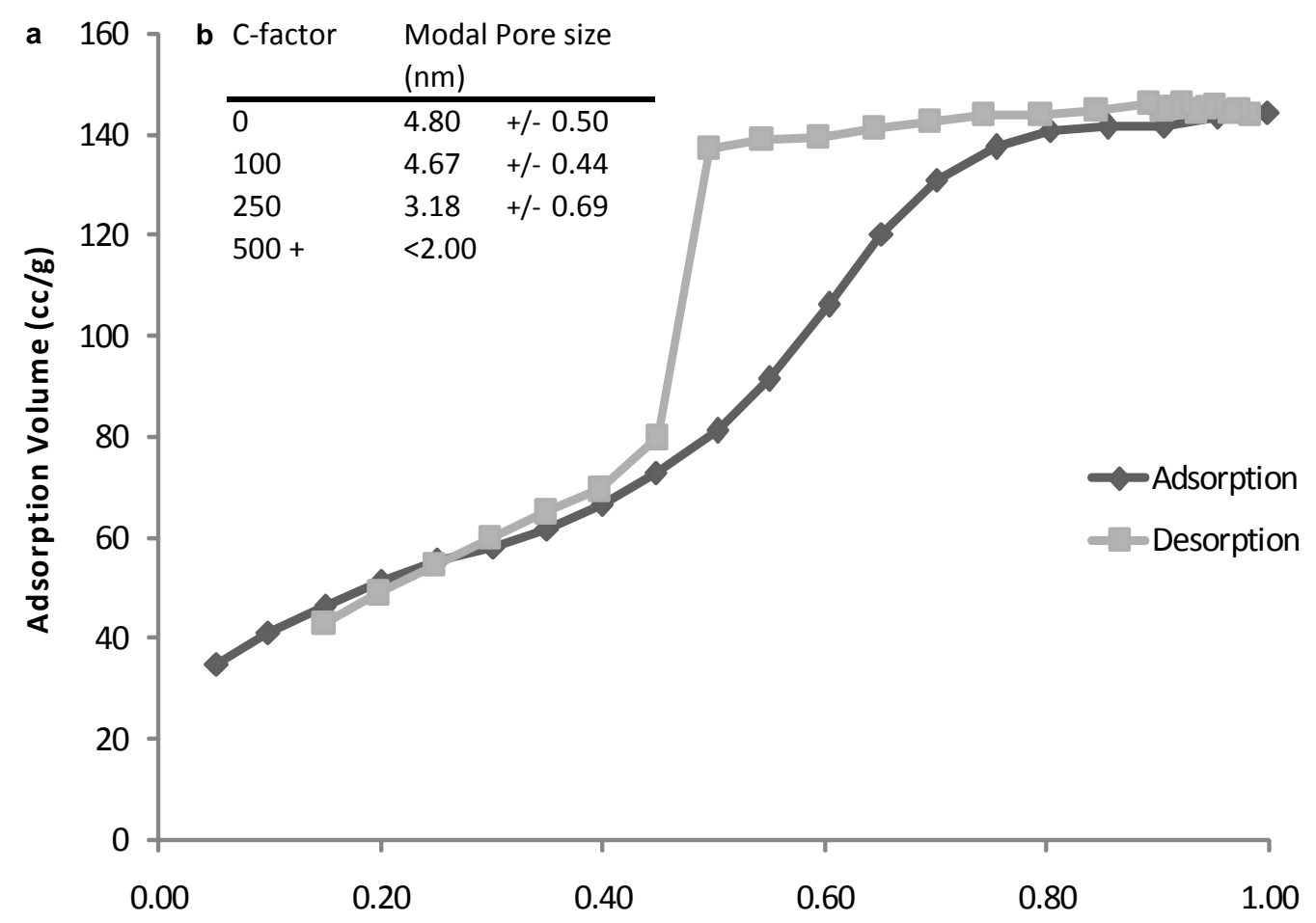

Relative Pressure (P/Po)

Figure 3. Mesopore characterisation of hybrids. a, a type IV adsorption/desorption isotherm for a hybrid (30 wt\% gelatin) of C-factor 100 acquired through nitrogen adsorption analysis. $\mathbf{b}$, the relationship between $\mathbf{C}$-factor and ( $30 \mathrm{wt} \%$ gelatin) hybrid modal pore size (+/- standard deviation).

SEM images reveal a texture resulting from fused nano particles, which are characteristic of mesoporous sol-gel silica (Fig. 4a and b). AFM was utilised in tapping mode to assess the surface properties of the hybrids (Fig. $4 \mathrm{c}$ and d). Phase imaging revealed stark contrasts between hybrids of high and low C-factors. The light regions in Figure 4 are representative of the organic phase ( $30 \%$ of the area for both Fig. $4 \mathrm{c}$ and 2 d) and the dark regions show the inorganic phase. The phase domain widths of C-factor 0 (Fig. 4c) were $90.6+/-9.5 \mathrm{~nm}$, over twice the size of those of C-factor 1500 (Fig. 4d), which were $44.3+/-6.1 \mathrm{~nm}$. This signified that the use of GPTMS as a coupling agent for the hybrids resulted in a much finer scale interaction of the 


\section{Submitted to \\ FUNCTIONAL}

constituent phases on the nanoscale. This correlates well to observations that hybrids of C-factors above 500 were transparent, while C-factors below 500 were progressively less transparent as quantified through colorimetric analysis

(Supplementary Fig. 1). The finer scale phase interaction shown in high C-factor materials arose as gelatin was functionalised with GPTMS prior to mixing with hydrolysed TEOS. Therefore the functionalised gelatin took part in the condensation reaction. In comparison, when non-functionalised gelatin (C-factor 0 ) was added to hydrolysed TEOS, the main mechanism for the gelatin interaction with the silicate network was through electrostatic attraction of the silicate non-bridging oxygens and amine groups of gelatin. ${ }^{[32]}$ This is a weaker interaction than covalent coupling with the GPTMS functionalised gelatin and hence constituent phase domains were larger. The NMR, AFM, FTIR and colorimetric data concur that covalent linkages were created between the silica and gelatin and that the degree of inorganic-organic coupling could be controlled. Next, it was important to determine whether the degree of coupling could be used to tailor the hybrid properties. 


\section{Submitted to}

a

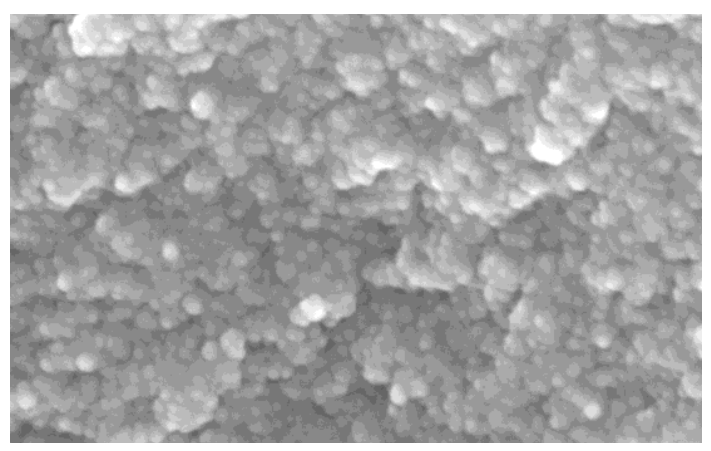

C

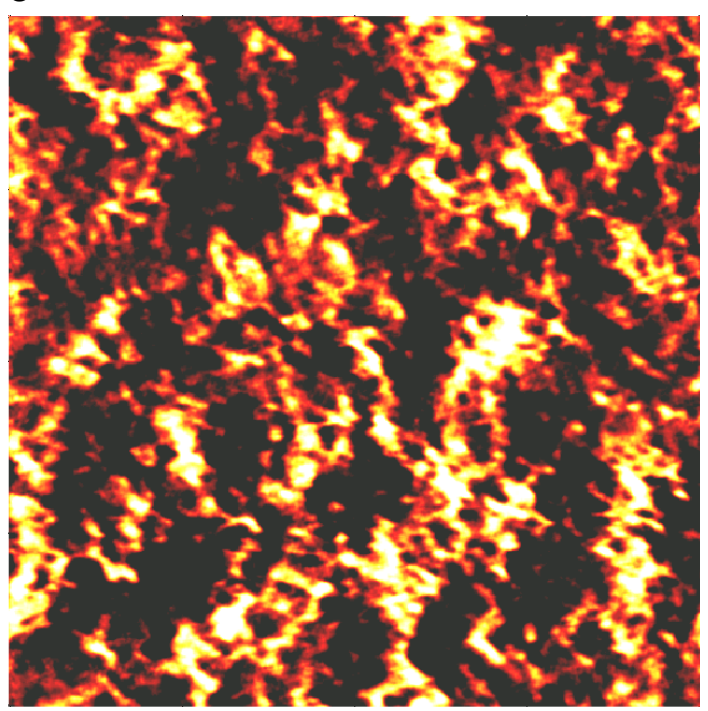

e C-factor Gelatin

\begin{tabular}{llll} 
& $($ wt\% $)$ & $(\mathrm{nm})$ \\
\hline 0 & 30 & $1.29+/-0.10$ \\
500 & 30 & $2.41+/-0.08$ \\
1500 & 30 & $5.33+/-0.12$ \\
1500 & 60 & $4.32+/-0.16$
\end{tabular}

Figure 4. Nano-textured hybrids. SEM images showing nanoscale texture of hybrids (30 wt\% gelatin) of C-factor 0 (a) and 1500 (b). Tapping mode AFM phase images for hybrids (30 wt\% gelatin) of C-factor 0 (c) and 1500 (d). Light regions signify the organic, whilst the darker regions represent the inorganic phase. Both scale bars are $200 \mathrm{~nm}$. e, the root-mean-square roughness (mean +/- standard deviation) of the hybrids, which increased with wt\% and C-factor. 


\subsection{Tailorable hybrid properties}

Submitted to

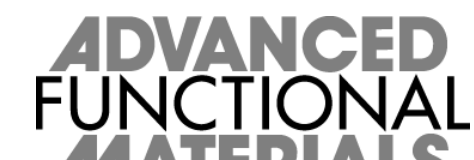

Resorbable biomaterials should exhibit controlled degradation so that mechanical properties can be maintained until the role performed by the biomaterial can be gradually transferred back to the newly formed tissue growing in its place. ${ }^{[33]}$ To test the dissolution properties of the hybrids the release of silicon and gelatin into simulated body fluid ${ }^{[34]}$ was monitored over time. As expected the gelatin release rate into solution decreased as the C-factor increased (Fig. 5a). For hybrids with Cfactor 100, the effect of GPTMS crosslinking was minimal so gelatin release was rapid with approximately $40 \%$ of the total gelatin content lost within 2 days, which was similar to hybrids with C-factor 0 (effectively a conventional composite as there is no covalent inorganic - organic link). In contrast, the C-factor 1500 hybrid released a negligible amount of gelatin over the duration of the entire study (20 days). Importantly however, the silicon release profiles (Fig. 5b) show similar trends to the gelatin release profiles. For hybrids with C-factors of 1500 the silicon release was

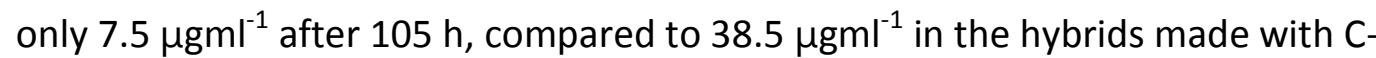
factor of 100 in the same time. The slower silica dissolution rate associated with increased GPTMS content is likely to be a result of the more highly condensed silicate network (shown with NMR, Fig. 1), as there were more Si-O-Si (bridging oxygen) bonds and fewer non-bridging oxygen bonds (Fig. 2). In summary, the addition of GPTMS in this system controlled the dissolution characteristics of both phases, leading to a material that degrades as one material rather than two independent phases. This is a considerable improvement on the degradation behaviour of conventional composites. 


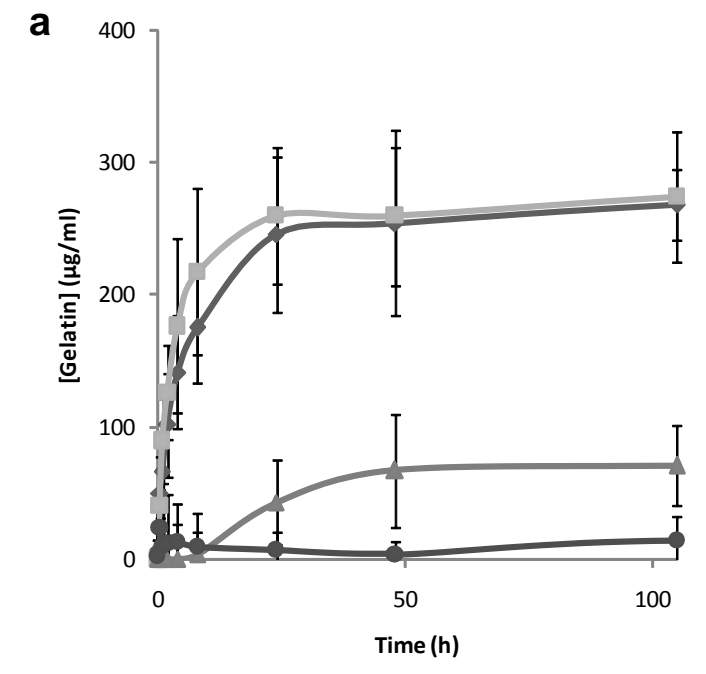

\section{Submitted to}

\section{FUNCANCED}

b

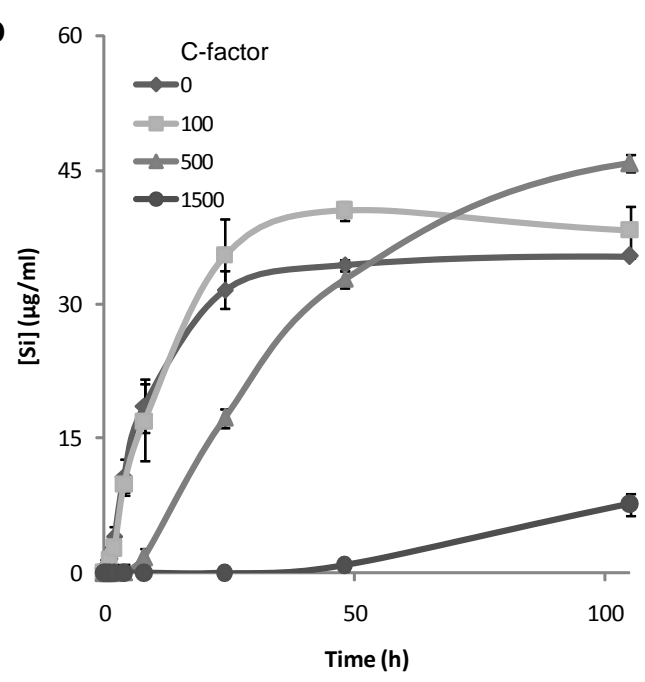

Figure 5. Release profiles of gelatin and silicon for hybrids ( $30 \mathrm{wt} \%$ gelatin) with Cfactor 0-1500 immersed in simulated body fluid (SBF). a, Gelatin concentration in SBF as measured by a protein assay (BCA). $\mathbf{b}$, The silicon concentration in SBF measured using ICP. Error bars represent the standard deviation.

For the hybrids to fulfil the criteria for a tissue regenerating construct they must be fabricated to form tough scaffolds with an open interconnected pore network and tailorable mechanical properties. The hybrid synthesis process was modified by adding a foaming step prior to gelation, similar to that previously developed by us for bioactive glass scaffold production. ${ }^{[35]}$ The foaming process involved the addition of surfactant to lower the surface tension of the sol and stabilise bubble formation during vigorous mixing before the foam gelled. A key development to the established foaming procedure was to incorporate a new freeze-drying step to remove the condensation by-products. The novel combination of sol-gel foaming, the inorganic-organic coupling reaction and freeze-drying produced nanostructured porous scaffolds with large pore networks (pore interconnect diameters greater than $200 \mu \mathrm{m}$ (Fig. 6). These scaffolds exhibit near spherical pore geometry rather than needle shaped pores typically associated with freeze-dried scaffolds. The spherical 


\section{Submitted to}

shapes of the pores were attributed to the foaming process prior to freeze drying,

which controls the size of these pores. As this pores are spherical not needle shaped

there are fewer areas of high stress concentration and therefore it forms stronger

scaffolds. The pore size can be tailored by controlling the foaming parameters such

as surfactant concentration. ${ }^{[36]}$ Freeze drying resulted in $100 \%$ crack-free scaffolds.

These scaffolds were less brittle than their oven dried counterparts because they

were subjected to less shrinkage throughout the drying process.

a

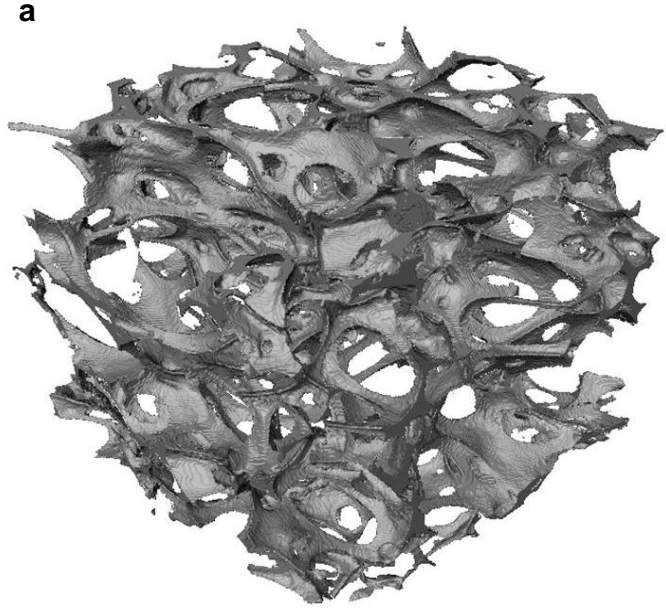

b

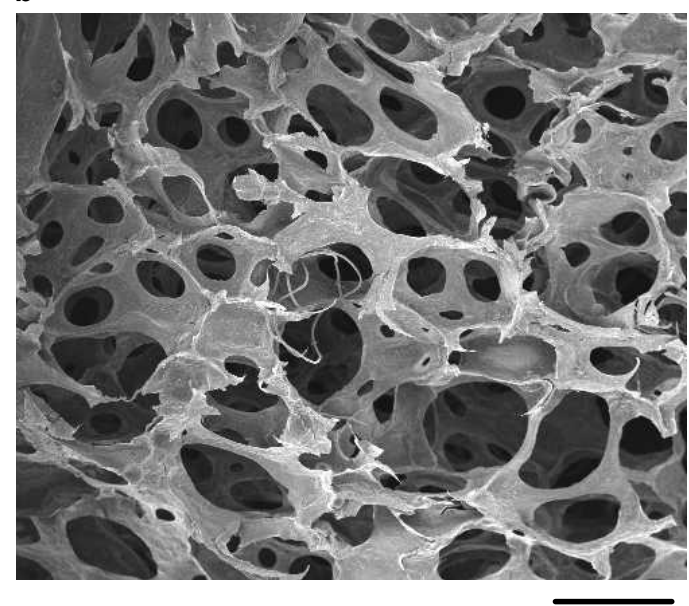

Figure 6. Hybrid foamed scaffold for tissue regeneration (30 wt\% gelatin with Cfactor 1000). a, X-ray micro-computed tomography image of the hybrid scaffold (courtesy of Sheng Yue, Department of Materials, Imperial College London). b, SEM image of the hybrid scaffold. The scale bar is $500 \mu \mathrm{m}$ for both. The images show a scaffold with an interconnected highly porous architecture generated from a sol-gel foaming and freeze drying technique.

By varying the percentage of gelatin and the degree of GPTMS crosslinking, the mechanical properties of the foam scaffolds could be tailored, as demonstrated here using compression testing (Fig. 7). Modal pore interconnect diameters of all scaffolds tested were $\sim 200 \mu \mathrm{m}$ (from mercury intrusion porosimetry, Fig. 7d), which is twice 
Submitted to

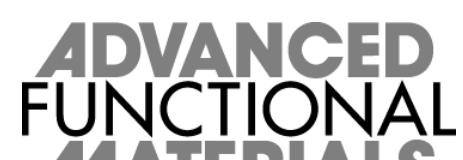

the minimum that is suggested to be necessary for vascularised tissue ingrowth. ${ }^{[37]}$

Depending on the inorganic and GPTMS content, the stiffness changed dramatically as shown by the stress strain response in Figure 7a. Scaffolds made with 60 wt\% gelatin and a C-factor of 500 were very flexible and highly elastic (Fig. 7c). The stressstrain response was similar to that of a thermoplastic polymer, with a linear-elastic region up to $34 \%$ strain before the pores became compressed and apparent stiffness increased (Fig. 7a). No yield point was observed. These properties would be highly suited to soft tissue applications where the material needs to withstand many cycles of low loads. Doubling the inorganic-organic coupling caused a $360 \%$ increase in stiffness (Fig. 7d). However, there was still a strain to failure of greater than $9 \%$, compared to $2 \%$ for non-organic containing bioactive glass scaffolds (Fig. $7 \mathrm{~b}),{ }^{[38]}$ demonstrating improved toughness through polymer addition. Systematically reducing the gelatin content was shown to produce stiffer materials, with stepped stress-strain curves attributed to micro fractures occurring at high stresses. Even with gelatin contents as low as $40 \%$, hybrids did not fail catastrophically. Hybrid scaffolds are compared to $100 \%$ inorganic bioactive glass scaffolds in Figure $7 \mathrm{~b}$. Catastrophic failure of the $100 \%$ bioactive glass scaffold, at less than $2.5 \%$ strain, resulted in immediate loss of strength to less than $30 \%$ of its maximum. Instead, hybrid scaffolds all maintained their maximum strength throughout failure, attributed to plastic deformation of the hybrid after yield. As a result, hybrid scaffolds are less likely to lose mechanical function instantaneously in vivo as they degrade. Further studies into the variation of mechanical properties throughout the hybrid degradation process will provide the basis for future studies. Most foam 


\section{Submitted to \\ FUNCTIONAL}

scaffolds were aged at room temperature, but also shown in Fig. 7a is the stress-

strain response for a scaffold aged at $40{ }^{\circ} \mathrm{C}$ (Fig. 7a C-factor 1000, 60 wt\%), which

exhibited an increased strength and stiffness of over $200 \%$, with little change in

strain to failure (Fig. 7d), indicating that ageing conditions play a significant role in

affecting strength. This study shows that elastic hybrids can be created with a range

of mechanical properties: stiffnesses close to those of glasses and to those of

polymer foams. As an indication, the stiffness ranges for biological tissues previously

measured have been indicated in Figure $7 a$, demonstrating the range of properties

tissue engineering constructs are required to match. ${ }^{[39-44]}$ 

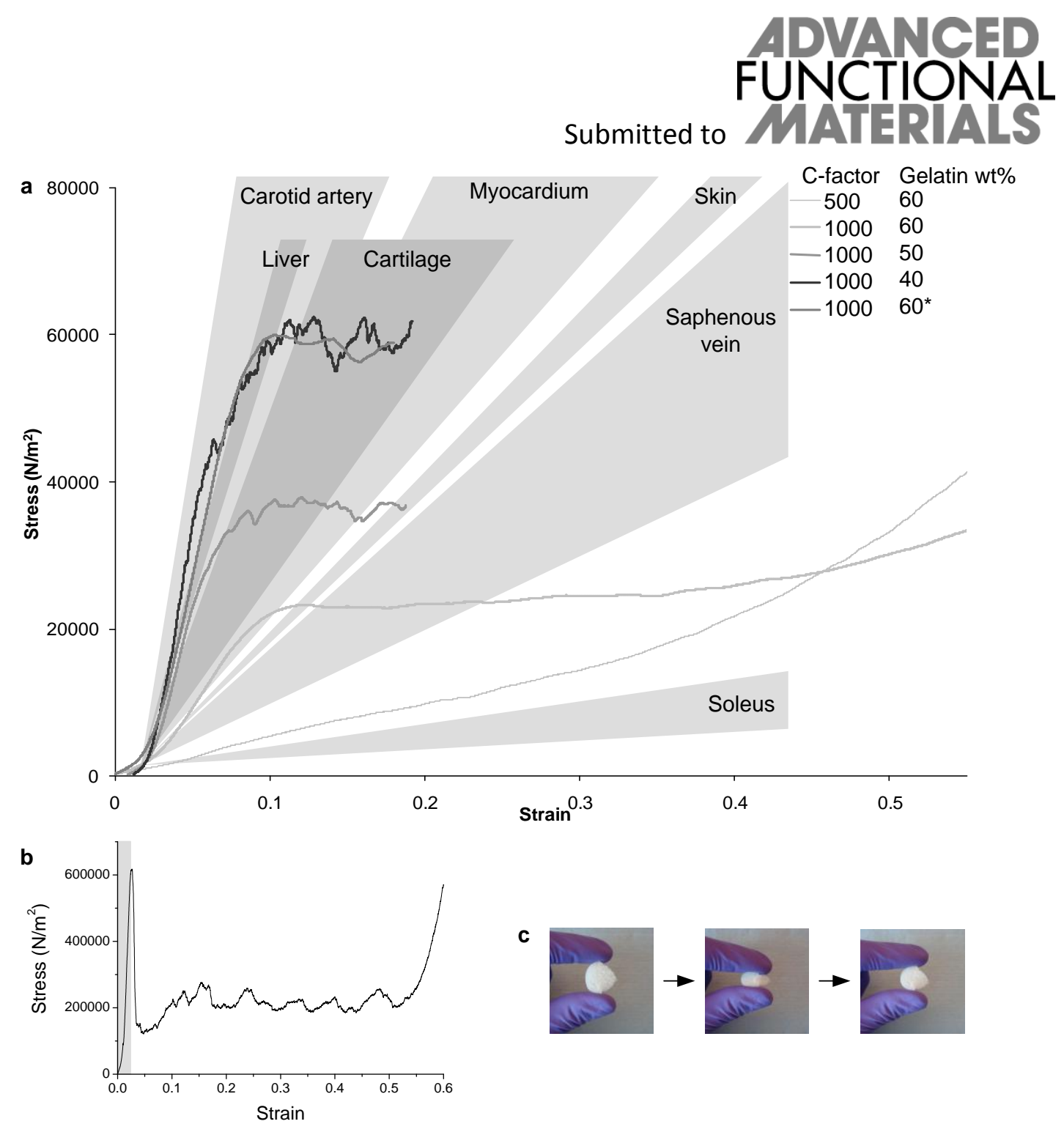

C

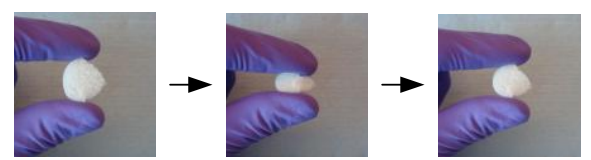

d C-factor Gelatin

Modal pore interconnect diameter $(\mu \mathrm{m})$

\begin{tabular}{llrrrr}
\hline 500 & 60 & $217.04+/-7.21$ & $19.03+/-5.10$ & $92.41+/-11.11$ & $34.1+/-3.46$ \\
1000 & 60 & $209.41+/-8.69$ & $23.25+/-1.61$ & $330.98+/-15.84$ & $8.84+/-0.78$ \\
1000 & 50 & $216.18+/-6.07$ & $32.24+/-3.13$ & $581.50+/-23.27$ & $7.79+/-0.84$ \\
1000 & 40 & $193.75+/-8.70$ & $60.40+/-5.05$ & $1266.60+/-68.66$ & $5.20+/-0.62$ \\
1000 & $60^{*}$ & $210.59+/-13.9$ & $53.85+/-6.54$ & $891.85+/-58.64$ & $8.88+/-0.47$
\end{tabular}

Figure 7. The mechanical properties of hybrid foam scaffolds, demonstrating the tailorable strength and elasticity. a, stress/strain curves for hybrids, showing an increasing stiffness, from highly elastic to stiff properties as C-factor and inorganic content was increased. Also indicated are the approximate stiffnesses of various tissues measured previously. ${ }^{[27-32]}$ These stiffness values are there only as a guide as most biological tissues exhibit viscoelastic properties, therefore their stress strain response is heavily dependent on the conditions of testing. $\mathbf{b}$, stress-strain response for a bioactive glass foam scaffold showing a brittle catastrophic failure mechanism at 0.025 strain (linear region is indicated by shaded portion), with no plastic 


\section{Submitted to

deformation after yield. c, Series of pictures demonstrating the highly elastic behaviour of C-factor 500 hybrid. $\mathbf{d}$, Scaffold properties, mean values +/- standard deviation. *indicates hybrid was aged at $40{ }^{\circ} \mathrm{C}$ rather than at room temperature.

In an attempt to optimise the hybrid foam strength whilst, maintaining the nonbrittle characteristics of the material, a hybrid composition of 53 wt\% gelatin, with a C-factor of 750 was synthesised. The degree of porosity was found to strongly influence the mechanical strength of the foam in accordance with cellular solid theory. ${ }^{[45]}$ Therefore hybrids with a range of porosities were synthesised by controlling the surfactant concentration during foaming. According to cellular solid theory

4

$$
E=E_{s}\left(\frac{\rho}{\rho_{s}}\right)^{2}
$$

where $E$ is the Young's modulus of the foam, $E_{s}$ is the Young's modulus of skeletal material, $\rho$ is the bulk density of the foam and $\rho_{s}$ is the density of the skeletal material. The term $\rho / \rho_{s}$ is known as the relative density $\left(\rho_{r}\right)$ of the foam. If the measured foam modulus is plotted against the relative density on a log scale there should be a linear relationship with a gradient of 2 as $E \propto \rho_{r}^{2}$. Our data confirms this relationship (Fig. 8a) and so it can be assumed that foams behave according to the cellular solid theory.

The failure mode of the foams is by plastic collapse, where the foam walls deform due to local moments, which exceed their fully plastic moment. According to cellular solid theory,

$$
\sigma_{p l}=0.3 \sigma_{y}\left(\frac{\rho}{\rho_{s}}\right)^{3 / 2}
$$




\section{Submitted to

where $\sigma_{p l}$ is the foam plastic collapse strength and $\sigma_{y}$ is the skeletal yield strength. ${ }^{[45]}$

By calculating $\sigma_{y}$ from the experimentally acquired plastic collapse strengths we find a mean skeletal yield strength of $117+/-8.05 \mathrm{MNm}^{-2}$. Based on this cellular solid model we can predict the plastic collapse strength of this composition of foam for any porosity (porosity $=1 / \rho_{r}$ ) as shown in Fig. $8 \mathrm{~b}$. It is also possible to relate porosity to the modal interconnect size based geometric assumptions and modal pore size data acquired from mercury intrusion porosimetry (Supplementary Fig. 2 and Method section 4.6). By showing the relationship between modal pore interconnect size, porosity and the plastic collapse strength it enables us to design foams based on the requirements for their application. For instance, if a modal pore diameter of $100 \mu \mathrm{m}$ is specified for vascularisation reasons, we can predict that a foam with this interconnect diameter will have a strength of $1.5 \mathrm{MNm}^{-2}$ (Fig. 8b). It is feasible to envisage that, with continued optimisation of composition and ageing conditions, strengths in excess of $2 \mathrm{MNm}^{-2}$ could be achieved, making scaffolds suitable for some bone tissue applications. ${ }^{[46]}$ Equally one could specify a foam strength for a desired application and predict the porosity or pore interconnect diameter that the foam would exhibit. 


\section{FUNCTIONAL}

a
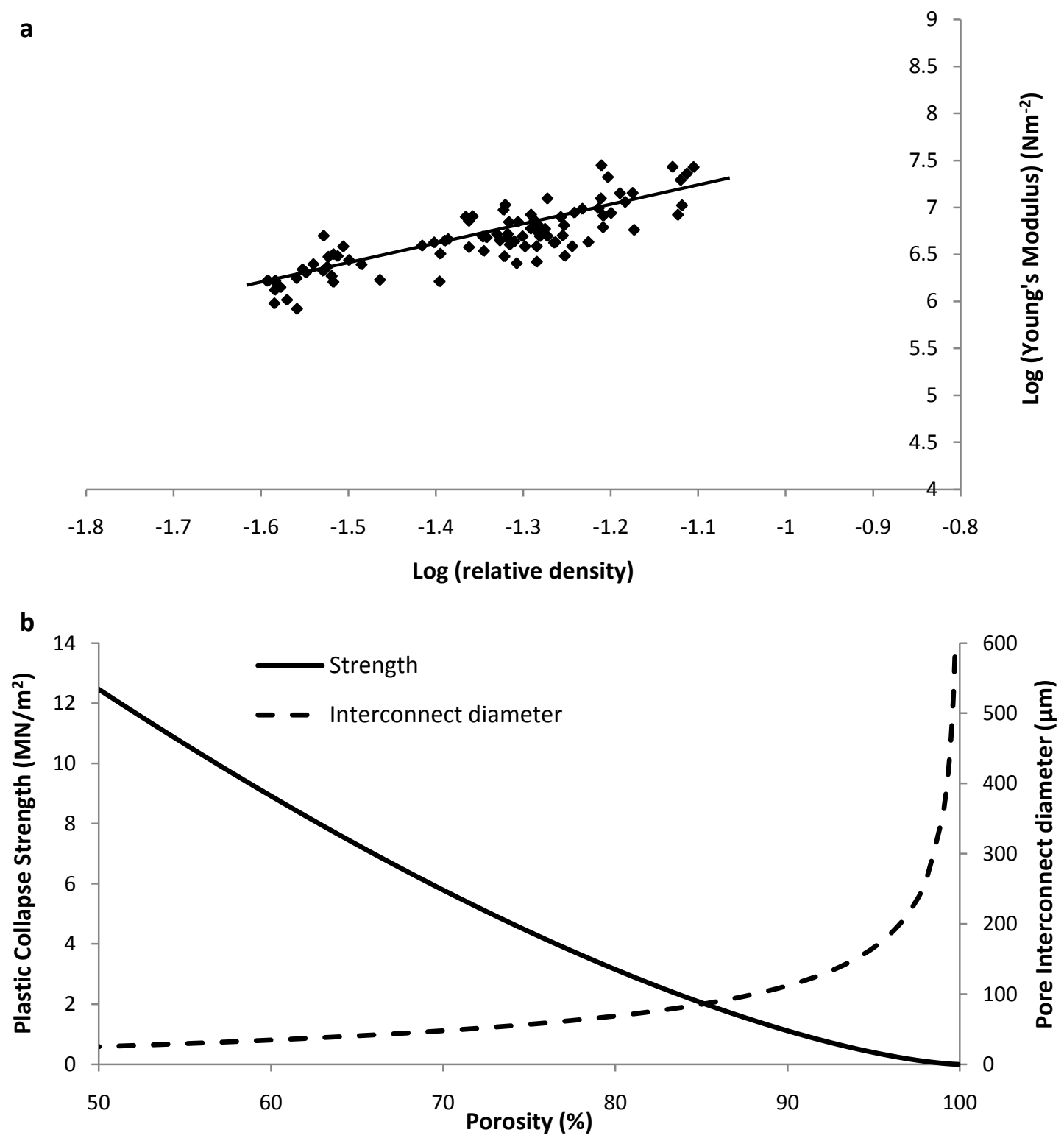

Figure 8. The relationship between mechanical properties and porosity for a hybrid foam of 53 wt\% gelatin and C-factor 750. a, A linear relationship with gradient of $2.04\left(R^{2}=0.88\right)$ between the log of relative density and Young's Modulus is shown indicating that foams are behaving according to cellular solid theory. $\mathbf{b}$, The predicted relationship between the plastic collapse strength and pore interconnect diameter based on cellular solid theory, also displaying on the right hand axis the relationship between porosity and pore interconnect diameter. 


\subsection{Biocompatible hybrids}

Submitted to

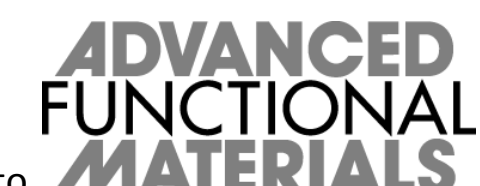

A potential risk with the low temperature synthesis method utilised in this study is that by-products, toxic to cells, could remain in the material. Therefore it was essential to consider the biocompatibility of the hybrids. Since the hybrids are intended for tissue regeneration, human bone marrow derived mesenchymal stem cells (MSCs) were used to investigate their cellular response. MSCs are multipotent, self-renewing stem cells that have an inherent potential to differentiate towards cell lineage of various types. ${ }^{[47]}$ They are promising in tissue engineering strategies as they can be harvested from the bone marrow of the patient and their potential to differentiate to bone, fat and cartilage has been extensively exploited in regenerative therapy. During tissue regeneration, MSCs are believed to egress away from their niche ${ }^{[48]}$ and migrate, engraft and differentiate within the tissue microenvironment. ${ }^{[49]}$ The LIVE/DEAD cytotoxicity assay carried out on the hybrids demonstrated that none of the compositions were toxic or induced cell death (red cells) after 7 days in culture (Fig. 9a). However, staining for live MSCs (green, Fig. 9a) revealed that cell attachment and morphology varied between the hybrids and seemed to be affected by C-factor. After 3 days the number of attached cells increased on hybrids as C-factor increased, although this trend was less apparent on the $30 \mathrm{wt} \%$ gelatin hybrids at the 7 day time point. This decrease in cell attachment was thought to be due to matrix elasticity, surface chemistry or topography of the 30 wt\% gelatin hybrids rather than toxicity as there was no evidence of dead cells. Indeed, the $30 \mathrm{wt} \%$ gelatin hybrids were found to be substantially smoother than their 60 wt\% counterparts through AFM RMS roughness values (Fig. 4e). In contrast, 


\section{FUNCTIONAL \\ submitted to 1 ATERILS}

the MSC attachment on $60 \mathrm{wt} \%$ gelatin hybrids increased with greater C-factor, but

also increased over time suggesting that MSCs proliferated on the $60 \mathrm{wt} \%$ hybrids

over the 7 day time period (Fig. 9a).
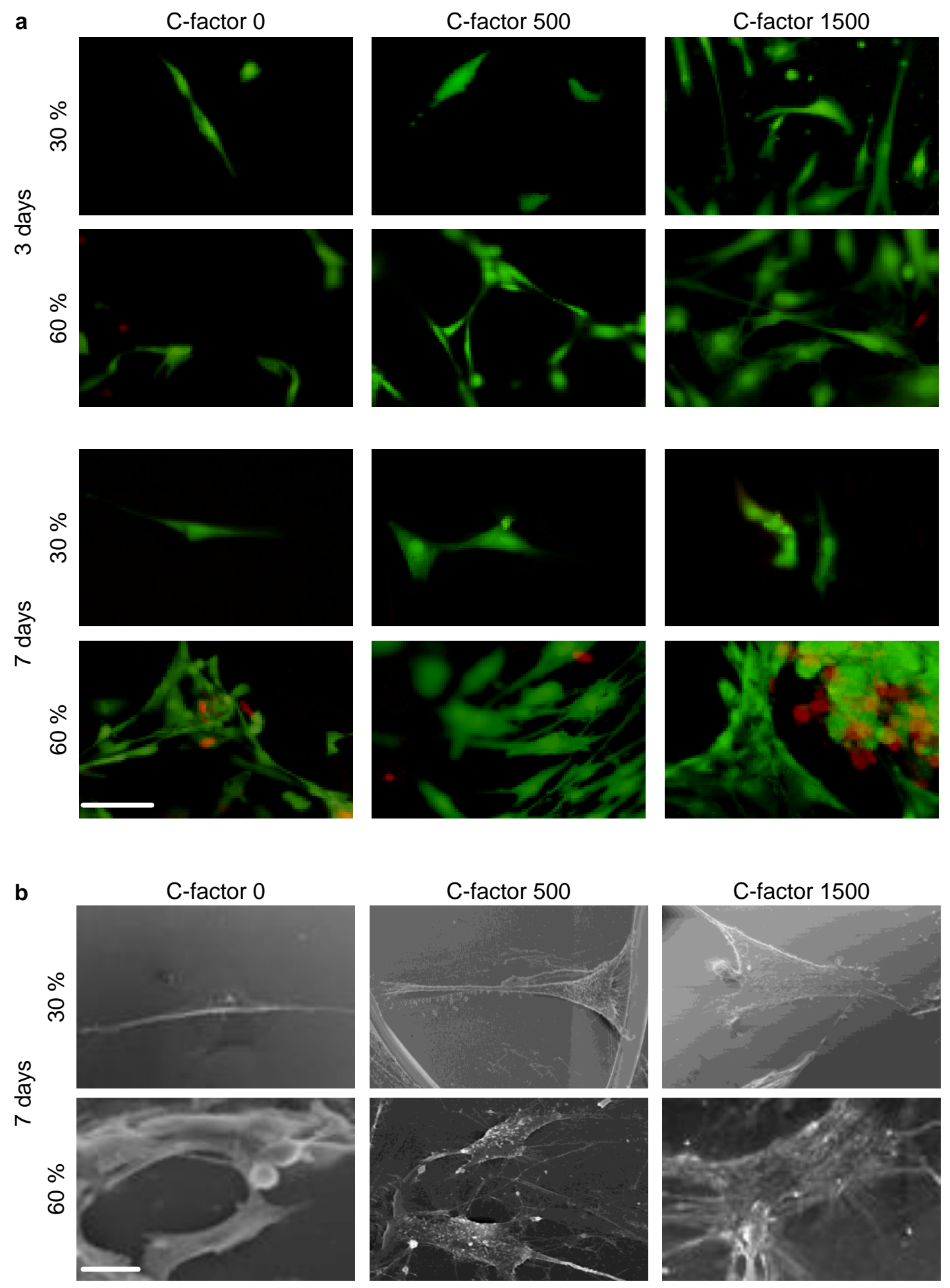


\section{Submitted to \\ FUNCTIONAL}

Figure 9. MSC viability and morphology following culture on monolith hybrids. a, In vitro cytotoxicity of the hybrids was determined by the LIVE/DEAD assay employing calcein AM for staining of the live cells (green) and ethidium homodimer-1 for labelling the nuclei of dead cells (red). (Scale bar $100 \mu \mathrm{m}$ ). b, SEM images of MSCs cultured on the hybrids for 7 days revealed different cell morphology on different hybrids (Scale bar=20 $\mathrm{mm}$ ).

Cell morphology was also found to vary depending on composition. Scanning electron microscopy (SEM) showed that MSCs on the 30 wt\% gelatin C-factor 0 hybrid were elongated spindle-shaped, with short filopodia-like extensions and limited branching (Fig. 9b). But with increasing C-factor and gelatin concentration cells were observed to spread and adhere better with increased branching filopodia. On the 60 wt\% gelatin C-factor 1500 hybrid, MSCs formed multicellular layers that appeared to deposit extracellular matrix protiens. [sentence removed]

Immunostaining of the vimentin and actin cytoskeleton across the hybrid series proved consistent with the phenotypical profiling demonstrated by SEM. In the cytoskeleton, vimentin is the major intermediate filament protein and actin is the most important interconnected filament protein that performs numerous critical cellular functions including maintaining cell shape, assisting cell movement and acting as tracks for organelle and enzyme movement within the cell. ${ }^{[50]}$ After 1 week on the $30 \mathrm{wt} \%$ gelatin hybrids vimentin appeared concentrated around the cell nucleus with little organisation on the C-factor 0 sample, but with increased C-factor, vimentin showed more mature extension throughout the cytoplasm (Fig. 10). The actin cytoskeleton was also immature for low C-factor hybrids, showing little organisation, with only diffuse actin throughout the MSCs. Stress fibres became 


\section{Submitted to

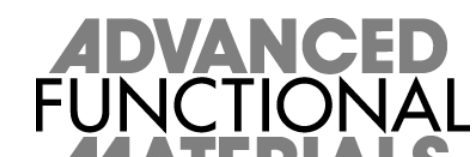

more defined as the C-factor of the hybrids increased. The organisation of the MSCs on the $60 \mathrm{wt} \%$ gelatin hybrids appeared to be more advanced with the cells demonstrating more defined vimentin fibres independent of C-factor. MSCs on $60 \mathrm{wt} \%$ C-factor 500 appeared to have a very well organised actin cytoskeleton with well defined stress fibres apparent on almost all cells (Fig. 10). Cell spreading and actin organisation also increased with increasing C-factor. Since one of the major roles assigned to vimentin is to provide support for the cells and the nucleus, the increasingly filamentous appearance of cells on the hybrids with higher C-factor could suggest increased mechanical stability of the cells on these hybrids. Although, the hybrid's effect on MSC differentiation was outside the scope of this study, it is worth considering that matrix stiffness, which has been shown to influence MSC differentiation to specific tissue lineages, ${ }^{[51]}$ is increased by higher C-factor (Fig. 7) and so may have contributed to the different cell morphologies and cytoskeleton characteristics observed. Whilst different hybrid compositions induced different cell morphologies, it is important to emphasise that all were shown to be biocompatible as they were non-cytotoxic. 


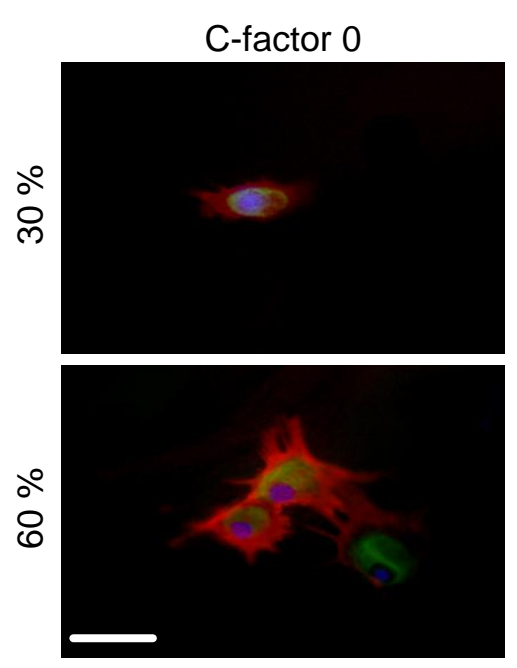

Submitted to

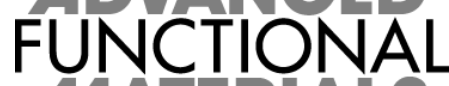

C-factor 500

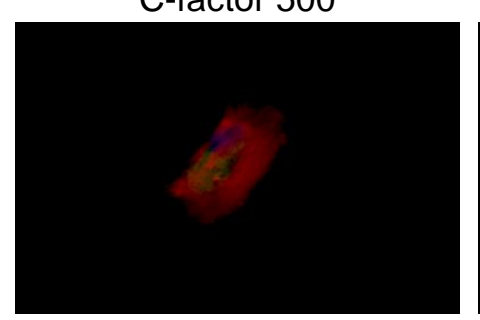

C-factor 1500
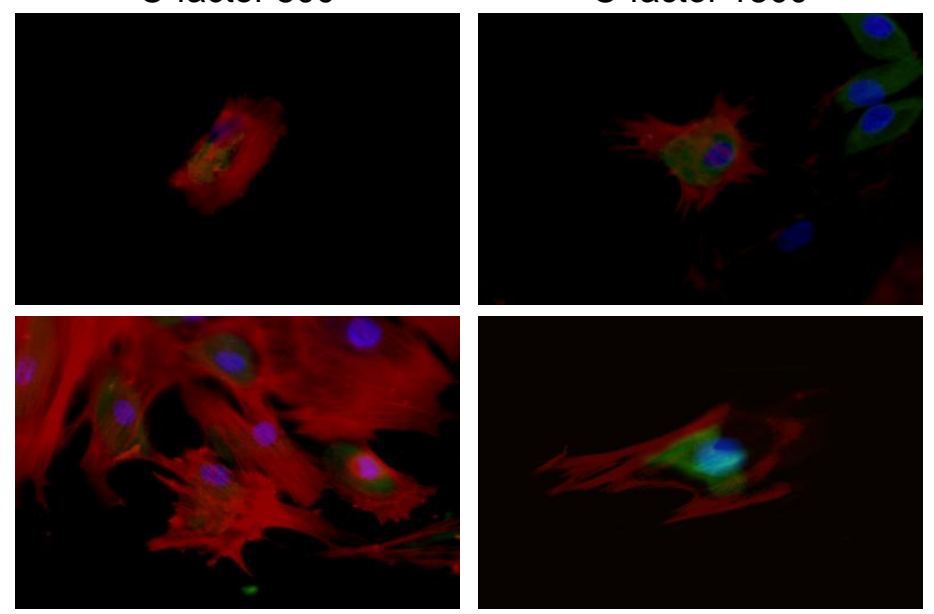

Figure 10. Fluorescence images of cells on the hybrids stained for the cytoskeletal proteins actin (red) and vimentin (green) and nuclei (blue). (Scale bar 100 $\mu \mathrm{m}$ )

We have engineered a new class of sophisticated silica-gelatin hybrid materials with

highly tailorable material properties. Exciting developments are easily envisaged

through further mechanical and biological optimisation of the scaffolds for individual hard and soft tissues, including the facile incorporation of biologics.

\section{Conclusions}

Tough biocompatible hybrids that can be fabricated into highly porous scaffold geometries using a novel sol-gel foaming and freeze drying approach have been developed. Hybrid scaffolds are intended to perform as a platform technology that can be applied to a range tissue of regeneration strategies. Essential to the success of these materials is their ability to be highly tailored to their application both in terms of mechanical properties and dissolution properties. We have shown that the inorganic-organic coupling is essential to provide a true nanoscale interaction between phases and providing enhanced control over material dissolution characteristics. The scaffold mechanical properties were also heavily influenced by 


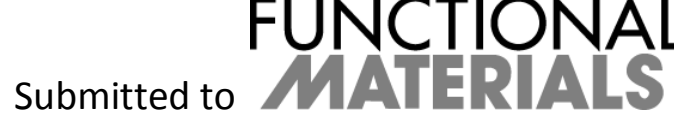

the degree of inorganic-organic coupling, together with the relative proportions of inorganic and organic present in the hybrid. Furthermore, the scaffold porosity was shown to directly influence the scaffold strength in a predictable manner allowing for the design of scaffolds with specific strengths, through control of their macroporosity.

\section{Experimental}

\subsection{Monolith synthesis}

All reagents were supplied by Sigma Aldrich UK unless otherwise specified. Initially gelatin (Bovine, Type A) was dissolved in $10 \mathrm{mM}$ hydrochloric acid $(\mathrm{HCl}$ ) at a concentration of $50 \mathrm{mgml}^{-1}$. This solution was functionalised by addition of an appropriate amount of GPTMS to give C-factors (the mole ratio of GPTMS/gelatin) ranging between 0-2000. The functionalised gelatin solution was left to mix for $14 \mathrm{~h}$ before being added as a sol-gel precursor.

A sol was prepared by adding in the following order: deionised water, $\mathrm{HCl}(1 \mathrm{~N})$ and tetraethyl orthosilicate (TEOS). The mole ratio of water/TEOS was 4 and $\mathrm{HCl}$ was added to catalyse TEOS hydrolysis at a volume ratio (water/HCl) of 3. The solution was stirred for 1 hour to allow hydrolysis of TEOS, before adding the functionalised gelatin solution, which was mixed for a further $1 \mathrm{~h}$. At higher $\mathrm{C}$-factors, the total organic content was increased substantially due to the organic contribution from GPTMS. Therefore rather than fix the gelatin content of the material it was decided, instead, to keep the volume ratio of functionalised gelatin added to the sol constant. 


\section{Submitted to FUNCTIONAL}

Two volume ratios (functionalised gelatin/hydrolysed TEOS) were used in this study:

$60 / 40$ and $85 / 15$ referred to as $30 \mathrm{wt} \%$ and $60 \mathrm{wt} \%$ respectively, representing the final weight percentages of gelatin in the $0 \mathrm{C}$-factor materials. Once the functionalised gelatin solution has mixed for 1 hour in the sol, hydrofluoric acid (HF, $5 \% \mathrm{v} / \mathrm{v}$ ) was added to the sol at sol/HF volume ratio of $100 / 1$ to catalyse the inorganic condensation reaction. $400 \mu$ laliquots of the sol were then transferred to 48 well micro plates for gelling and ageing. The gels were aged for 1 week and then dried at $60{ }^{\circ} \mathrm{C}$ for $120 \mathrm{~h}$.

\subsection{Foaming and freeze-drying}

To form scaffold structures from hybrid materials the same initial preparation for monolith synthesis was carried out until solutions of functionalised gelatin and hydrolysed TEOS had been mixed for $1 \mathrm{~h}$. At this point $50 \mathrm{ml}$ of sol was transferred to a $600 \mathrm{ml}$ polypropylene beaker and $1.5 \mathrm{ml}$ of Triton X-100 and $1 \mathrm{ml}$ of HF were added immediately before a period of vigorous mixing in order to induce a foam. At the point of gelling (after approximately 4 mins) the foams were poured into wide mouth $60 \mathrm{ml}$ polycarbonate screw cap containers (Nalgene). They were either aged at $4{ }^{\circ} \mathrm{C}$ for 5 days or for 1 day at $4{ }^{\circ} \mathrm{C}$ followed by 4 days at $40{ }^{\circ} \mathrm{C}$. Drying was carried out by freeze drying samples (Thermo Scientific Heto PowerDry LL1500) at a temperature of $-96{ }^{\circ} \mathrm{C}$ and a pressure of $0.13 \mathrm{mbar}$. 


\subsection{Monolith Characterisation}

Submitted to

\section{FUNCANCED \\ MATERIALS}

Following ageing and prior to drying, the transparency of the gels was assessed using a colorimetric absorption system (Anthos Microtiterplate photometer HT III) at 550 $\mathrm{nm}$ wavelength. 12 gels for each C-factor (0-2000) were analysed simultaneously in a 96 well micro plate. Fourier transform infrared spectroscopy (Perkin Elmer Spectrum 100) was carried out on all samples in the range $0-2000 \mathrm{~cm}^{-1}$, at a resolution of $4 \mathrm{~cm}^{-}$ ${ }^{1 .}{ }^{29}$ Si Magic Angle Spinning Nuclear Magnetic Resonance (MAS NMR) spectra of hybrids were acquired on a Varian InfinityPlus $300 \mathrm{MHz}$ spectrometer operating at 59.62 MHz. The ${ }^{29}$ Si spectra were collected using MAS of $4 \mathrm{kHz}$ with a 20 s recycle delay and pulses with a tip angle of $\sim 30^{\circ}$. The pulse delay was sufficient to prevent saturation. Chemical shifts were referenced externally to TMS at 0ppm. Atomic force microscopy (Ambios Technology, Q-scope) in tapping mode was performed on hybrids to acquire both phase and surface topography data over a $2 \times 2 \mu \mathrm{m}$ area. Analysis was carried out using a non-contact silicon cantilever tip (Ambios Techonology, NSC-16 PN-371300). Phase domain widths were measured with Image J (version 1.37). X-ray micro-computed tomography $(\mu \mathrm{CT})$ was used to image the sample non-destructively. The sample was scanned using a commercial $\mu \mathrm{CT}$ unit (Phoenix X-ray Systems and Services GmbH, Wunstorf, Germany) at $80 \mathrm{KeV}$ and 125 $\mathrm{mA}$, and at the resolution of a $5 \mu \mathrm{m}$ voxel. Characterisation of the hybrid's mesopores was performed with nitrogen adsorption (Quantachrome AS6, UK) and modal pore sizes were determined using the BJH method on Quantachrome software (Autosorb 1, version 1.52). Field emission gun scanning electron microscopy (LEO Gemini 1525 FEGSEM) of hybrid nanostructure was carried out on 
hybrids to image their nanoscale morphology, with an accelerating voltage of 5.00 $\mathrm{kV}$ and a working distance $\sim 7 \mathrm{~mm}$.

\subsection{Dissolution study}

Hybrids (200 mg) were immersed in $100 \mathrm{ml}$ simulated body fluid (SBF). ${ }^{\left[{ }^{34]}\right.}$ At each of the 20 time points throughout the 20 day study $1 \mathrm{ml}$ sample was removed and replenished with SBF. $1 \mathrm{ml}$ samples from each time point $(n=3)$ were analysed for gelatin concentration (Pierce Micro BCA protein assay, with gelatin solution standard $(n=3))$ and Si concentration using ICP (PerkinElmer Optima 2000DV, flow rate of 1.5 $\mathrm{mlmin}^{-1}$ and plasma power of $\left.1300 \mathrm{~W}(\mathrm{n}=3)\right)$.

\subsection{Scaffold compression testing}

The macroporous dimensions of the scaffold were determined through mercury intrusion porosimetry (Quantachrome, Pormaster 33). Pore modal interconnect diameters were calculated based on the Washburn equation using Quantachrome Poremaster for Windows, version $7.01(n=3)$. Compression testing (Instron 5866) was carried out on right parallel piped scaffolds with a width/thickness ratio of 2:1 $(n=5)$. A $500 \mathrm{~N}$ load cell was used for testing, with a compression extension speed of 0.5 $\mathrm{mmmin}^{-1}$.

\subsection{Correlation of pore interconnect diameter and relative density}

In order to correlate modal pore interconnect diameter to relative density of foams a model was used based on geometric considerations. The assumptions were made 


\section{Submitted to

that the pore interconnect diameter could vary between 0 and $\infty$ and the mass,

skeletal volume and therefore skeletal density remained constant and independent

of pore interconnect diameter. As the modal interconnect diameter is a length scale then

6

$$
d \propto \frac{1}{\rho_{r}^{3}}
$$

where $d$ is the modal pore interconnect diameter and $\rho_{r}$ is the relative density.

Therefore we can say that,

7

$$
\rho_{r}^{-1 / 3}=A d+B
$$

where $A$ and $B$ are constants. When $d=0$, the skeletal density, $\rho_{s}$, is equal to the bulk density, $\rho$, therefore $\rho_{r}=1$ as $\rho_{r}=\rho / \rho_{s}$. Therefore

8

$$
\rho_{r}^{-1 / 3}=A d+1
$$

The constant $A$ can be found experimentally (see Supplementary Fig. 2)

\subsection{Cell culture}

\subsubsection{Human bone marrow mesenchymal stem cells}

MSCs were purchased from Lonza (Lonza, UK) and maintained in low glucose Dublecco's Modified Eagle Medium (DMEM) supplemented with $10 \%$ (v/v) batch tested Fetal Bovine Serum (FBS), $50 \mathrm{Uml}^{-1}$ penicillin and $50 \mathrm{\mu gml}^{-1}$ streptomycin, $1 \%$ (v/v) L-glutamine (all from Invitrogen, UK). Monolith hybrids were sterilised by immersion in $70 \%$ Ethanol for 20 min followed by 20 min incubation in PBS and numerous washes with PBS to ensure the removal of any residual ethanol. For all 


\section{FUNCTIONAL \\ Submitted to 1/A ER L}

experiments MSCs were seeded at a density of 10,000 cells $/ \mathrm{cm}^{2}$ and cultured on the monoliths without preconditioning.

\subsubsection{LIVE/DEAD assay}

The LIVE/DEAD viability/cytotoxicity assay (Molecular Probes, UK) was performed according to the manufacturer's instructions. Stained samples were examined under an Olympus BX-URA2 fluorescence microscope. Images were captured using a Zeiss Axiocam digital camera and analysed using KS-300 software (Imaging Associates).

\subsubsection{Scanning electron microscopy}

10,000 cells $/ \mathrm{cm}^{2} \mathrm{MSC}$ were seeded and cultured on the hybrids for 7 days. Then cells were fixed in $2.5 \%(\mathrm{v} / \mathrm{v})$ glutaraldehyde in phosphate-buffered solution (PBS) for 40 min at $4{ }^{\circ} \mathrm{C}$ followed by dehydration through careful addition of a series of increasing concentrations of ethanol ( $25 \%, 50 \%, 70 \%, 90 \%$ and $100 \%(v / v)$ in $\left.\mathrm{dH}_{2} \mathrm{O}\right)$ for 5 min at room temperature. Finally the samples were critically dried by incubation in hexamethyldisilazane (HMDS) for a final 5 min and left to air dry in a fume cabinet. Dry samples were gold sputter coated and viewed using JEOL 5610LV Scanning Electron Microscope (JEOL, USA).

\subsubsection{Actin and vimentin immunostaining}

MSCs on hybrids were fixed in $4 \%(\mathrm{w} / \mathrm{v})$ paraformaldehyde in PBS, with $1 \%(\mathrm{w} / \mathrm{v})$ sucrose at $37^{\circ} \mathrm{C}$ for $20 \mathrm{~min}$, washed with PBS and permeabilized with buffered $0.5 \%$ Triton X-100 (10.3g sucrose, $0.292 \mathrm{~g} \mathrm{NaCl}, 0.06 \mathrm{~g} \mathrm{MgCl}_{2}, 0.476 \mathrm{~g}$ (4-(2-hydroxyethyl)-1- 
Submitted to

piperazine-ethanesulphonic acid) (HEPES), $0.5 \mathrm{ml}$ Triton X-100, in $100 \mathrm{ml}$ water; $\mathrm{pH}$

7.2) at $4{ }^{\circ} \mathrm{C}$ for $5 \mathrm{~min}$. Non-specific binding sites were blocked with $1 \%(\mathrm{w} / \mathrm{v})$ bovine serum albumin (BSA) in PBS at $37^{\circ} \mathrm{C}$ for 5 min and subsequently incubated for $1 \mathrm{~h}$ with a 1:200 concentration of anti-vimentin (monoclonal anti-human raised in mouse (Clone V9, Dako, UK)). Simultaneously, Alexa 568-conjugated phalloidin was added for the duration of this incubation (1:100, Molecular Probes, UK). Non-specific charges were neutralised with $0.5 \%(\mathrm{v} / \mathrm{v})$ Tween 20/PBS to minimise background labelling. A secondary fluorescein isothiocyanate-conjugated antibody, (1:50 in $1 \%$ (w/v) BSA/PBS, monoclonal rabbit anti-mouse (IgG), Vector Laboratories, UK) was added for $1 \mathrm{~h}\left(37^{\circ} \mathrm{C}\right)$ and given a final wash. Samples were mounted in Vectorshield with DAPI mountant for fluorescence (Vector Laboratories, UK), and viewed with an Olympus BX-URA2 fluorescence microscope. Images were captured using a Zeiss Axiocam digital camera and analysed using KS-300 software (Imaging Associates). Image manipulation in Adobe Photoshop was then used to superimpose the colour channels (vimentin in green, actin in red and nuclei in blue).

\section{Acknowledgements}

Julian R. Jones is a Royal Academy of Engineering EPSRC Research Fellow, and EPSRC are thanked for additional funding work (EP/E057098 and EP/E051669). Molly M. Stevens thanks ERC FPT grant naturale. Mark E. Smith thanks the University of Warwick, the Regional Development Agency (AWM) and the ERDF for partially funding the NMR infrastructure at Warwick. 


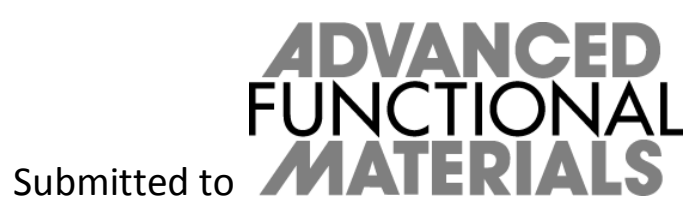

Received:

Revised:

Published online:

[1] E. Lavik and R. Langer, Appl. Microbiol. Biotechnol. 2004, 65, 1.

[2] M. M. Stevens and J. H. George, Science 2005, 310, 1135.

[3] M. P. Lutolf and J. A. Hubbell, Nat. Biotechnol. 2005, 23, 47.

[4] E. S. Place, N. D. Evans, M. M. Stevens, Nature Mater. 2009, 8, 457.

[5] S. Radin, G. El-Bassyouni, E. J. Vresilovic, E. Schepers, P. Ducheyne, Biomaterials 2005, 26, 1043.

[6] L. L. Hench and H. A. Paschall, J. Biomed. Mater. Res. 1973, 7, 25.

[7] L. L. Hench and J. Wilson, Science 1984, 226, 630.

[8] L. L. Hench and J. M. Polak, Science 2002, 295, 1014.

[9] K. A. Hing, L. E. Wilson, T. Buckland, Spine J. 2007, 7, 475.

[10] A. Leu and J. K. Leach, Pharm. Res. 2008, 25, 1222.

[11] I. I. Slowing, J. L. Vivero-Escoto, C. W. Wu, V. S. Y. Lin, Adv. Drug Delivery Rev. $2008,60,1278$.

[12] A. Moshaverinia, N. Roohpour, T. U. Rehman, Acta Biomater. 2009, 5, 498.

[13] Q. Z. Z. Chen, I. D. Thompson, A. R. Boccaccini, Biomaterials 2006, 27, 2414.

[14] S. Heinemann, H. Ehrlich, C. Knieb, T. Hanke, Int. J. Mater. Res. 2007, 98, 603.

[15] M. Wang, Biomaterials 2003, 24, 2133.

[16] G. Kickelbick, Prog. Polym. Sci. 2003, 28, 83.

[17] B. M. Novak, Adv. Mater. 1993, 5, 422. 


\section{submitted to}

[18] J. F. Guo, L. S. Fu, H. R. Li, Y. X. Zheng, Q. G. Meng, S. B. Wang, F. Y. Liu, J. Wang, H. J. Zhang, Mater. Lett. 2003, 57, 3899.

[19] R. L. Guo, C. L. Hu, F. S. Pan, H. Wu, Z. Y. Jiang, J. Membr. Sci. 2006, 281, 454.

[20] F. Li, X. M. Li, S. S. Zhang, J. Chromatogr. A 2006, 1129, 223.

[21] G. Falini, S. Fermani, E. Foresti, B. Parma, K. Rubini, J. Mater. Chem. 2004, 14, 2297.

[22] J. Heino, Bioessays 2007, 29, 1001.

[23] I. Grizzi, H. Garreau, S. Li, M. Vert, Biomaterials 1995, 16, 305.

[24] T. Coradin, S. Bah, J. Livage, Colloids Surf. , B 2004, 35, 53.

[25] S. Smitha, P. Shajesh, P. Mukundan, T. D. R. Nair, K. G. K. Warrier, Colloids Surf. , B 2007, 55, 38.

[26] S. Smitha, P. Mukundan, P. K. Pillai, K. G. K. Warrier, Mater. Chem. Phys. 2007, 103, 318.

[27] L. Ren, K. Tsuru, S. Hayakawa, A. Osaka, J. Sol-Gel Sci. Technol. 2001, 21, 115.

[28] L. Ren, K. Tsuru, S. Hayakawa, A. Osaka, Biomaterials 2002, 23, 4765.

[29] L. L. Hench and J. K. West, Chem. Rev. 1990, 90, 33.

[30] S. Lin, C. Ionescu, K. J. Pike, M. E. Smith, J. R. Jones, J. Mater. Chem. 2009, 19, 1276.

[31] B. Riegel, W. Kiefer, S. Hofacker, G. Schottner, J. Sol-Gel Sci. Technol. 2002, 24, 139.

[32] S. Heinemann, C. Heinemann, H. Ehrlich, M. Meyer, H. Baltzer, H. Worch, T. Hanke, Adv. Eng. Mater. 2007, 9, 1061.

[33] L. G. Griffith and G. Naughton, Science 2002, 295, 1009. 


\section{Submitted to

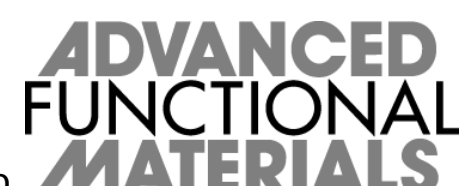

[34] T. Kokubo, H. Kushitani, S. Sakka, T. Kitsugi, T. Yamamuro, J. Biomed. Mater. Res. 1990, 24, 721.

[35] P. Sepulveda, J. R. Jones, L. L. Hench, J. Biomed. Mater. Res. 2002, 59, 340.

[36] J. R. Jones and L. L. Hench, J. Mater. Sci. 2003, 38, 3783.

[37] S. F. Hulbert, S. J. Morrison, J. J. Klawitte, J. Biomed. Mater. Res. 1972, 6, 347.

[38] J. R. Jones, L. M. Ehrenfried, L. L. Hench, Biomaterials 2006, 27, 964.

[39] S. Bensamoun, L. Stevens, M. J. Fleury, G. Bellon, F. Goubel, M. C. H. B. Tho, J. Biomech. 2006, 39, 568.

[40] Q. Z. Chen, S. E. Harding, N. N. Ali, A. R. Lyon, A. R. Boccaccini, Mat. Sci. Eng. R 2008, 59, 1.

[41] G. Constantinides, Z. I. Kalcioglu, M. McFarland, J. F. Smith, K. J. Van Vliet, J. Biomech. 2008, 41, 3285.

[42] Y. C. Fung, in Biomechanics: Mechanical Properties of Living Tissues 2nd ed., Springer-Verlag, New York, USA 1993, pp. 346-

[43] R. K. Korhonen, M. S. Laasanen, J. Toyras, J. Rieppo, J. Hirvonen, H. J. Helminen, J. S. Jurvelin, J. Biomech. 2002, 35, 903.

[44] R. L. Wesly, R. N. Vaishnav, J. C. Fuchs, D. J. Patel, J. C. Greenfield, Jr., Circ. Res. $1975,37,509$.

[45] L. J. Gibson, M. F. Ashby, in Cellular solids: structure and properties 2nd ed., Cambridge University Press, Cambridge 1999, pp. 175-231

[46] D. R. Carter and W. C. Hayes, Science 1976, 194, 1174. 


\section{Submitted to}

[47] M. F. Pittenger, A. M. Mackay, S. C. Beck, R. K. Jaiswal, R. Douglas, J. D. Mosca, M. A. Moorman, D. W. Simonetti, S. Craig, D. R. Marshak, Science 1999, 284, 143.

[48] Y. Katayama, M. Battista, W. M. Kao, A. Hidalgo, A. J. Peired, S. A. Thomas, P. S. Frenette, Cell 2006, 124, 407.

[49] R. Okamoto, T. Yajima, M. Yamazaki, T. Kanai, M. Mukai, S. Okamoto, Y. Ikeda, T. Hibi, J. Inazawa, M. Watanabe, Nat. Med. 2002, 8, 1011.

[50] D. E. Ingber, Cell 1993, 75, 1249.

[51] A. J. Engler, S. Sen, H. L. Sweeney, D. E. Discher, Cell 2006, 126, 677.

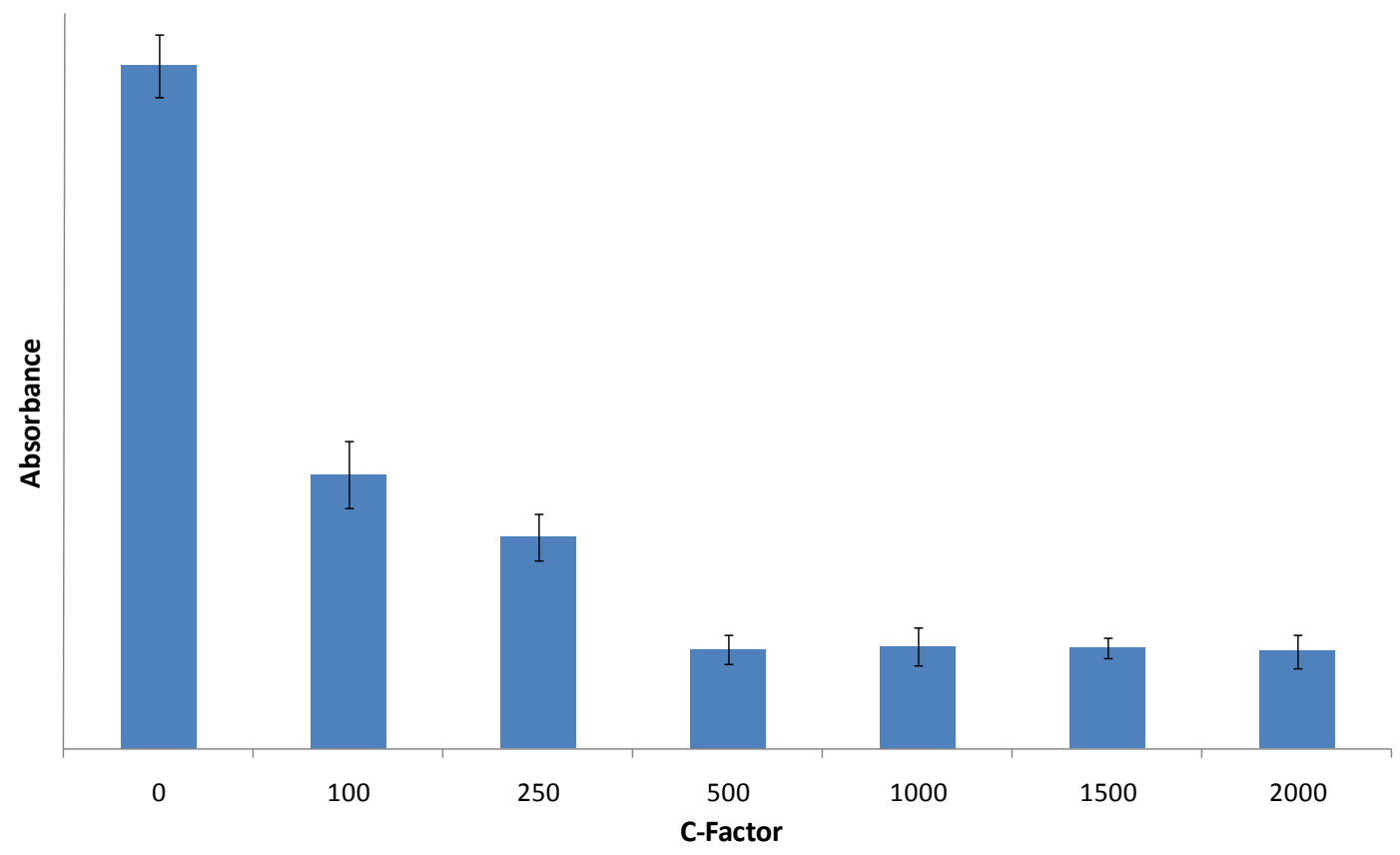

Supplementary Figure 1. Light absorbance of hybrids gels ( $30 \mathrm{wt} \%$ gelatin) prior to drying at a wavelength of $550 \mathrm{~nm}$. A higher absorbance indicates lower transparency of gels. Transparency of the hybrid gels increased as C-factor increased due to the combined effect of better inorganic-organic phase integration and a reduction in mesoporosity. Error bars indicate standard deviation 


\section{FUNCTIONAL \\ Submitted to}

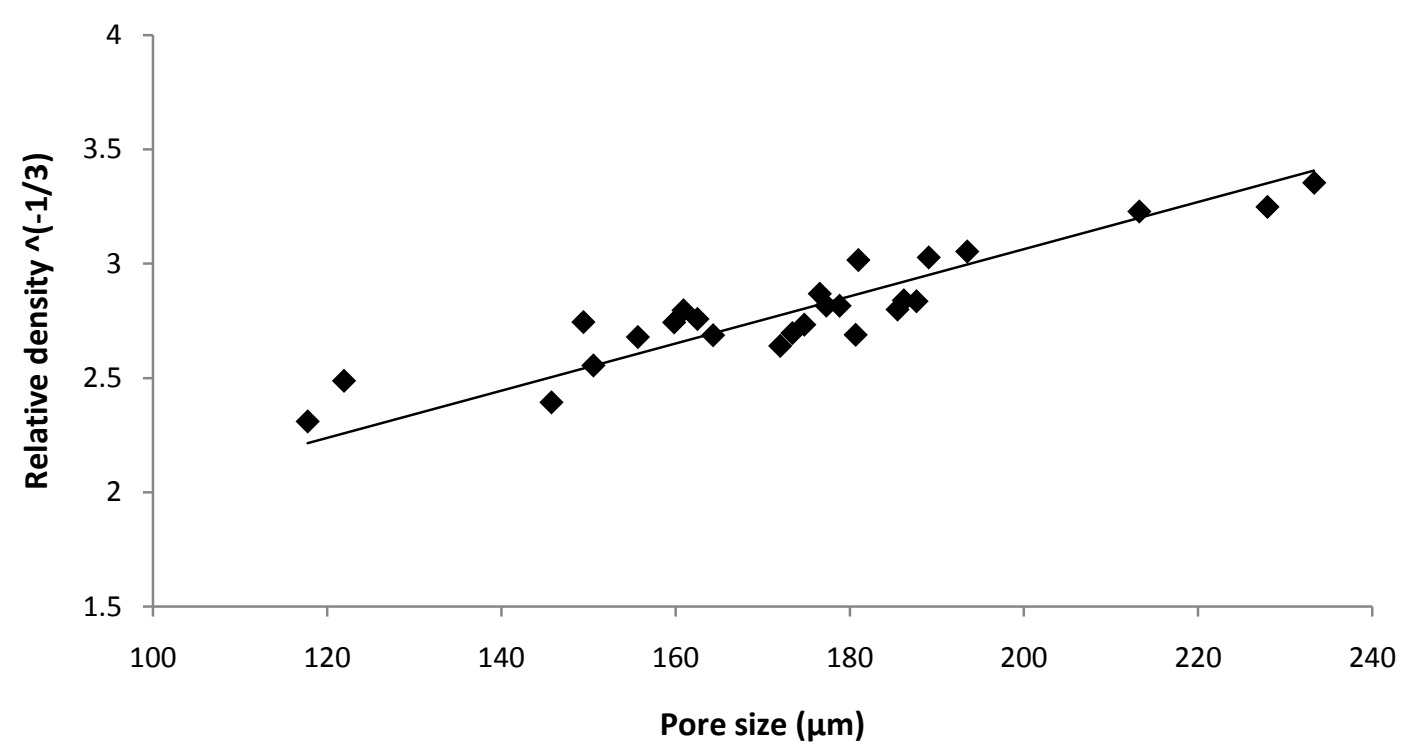

Supplementary Figure 2. Relative density shown as a function of the modal pore interconnect diameter found experimentally for a hybrid with $53 \mathrm{wt} \%$ gelatin and Cfactor 750. The data was fitted to the line $\rho_{r}^{-1 / 3}=0.0103 d+1\left(R^{2}=0.86\right)$. This relationship was used to correlate pore interconnect diameter to foam strength 